\title{
PERGEPTION OF MOVING FIGURES 1, ON THE REDUCTION PHENOMENA
}

\author{
YOSHIHISA TANAKA AND YOSHIAKI NAKAJIMA
}

University of Tokyo

\begin{abstract}
An oblong rectangle moving horizontally shows decrease in its perceived length parallel to the moving difrection, when the moving speed increases up to a certain point. Present study concerns with the quantitative relationship between the perceived size of a moving figure and its moving speed.

A black rectangle projected on a screen moves horizontally. The speed ranges from $32.2 \% \mathrm{sec}$ to $120.6^{\circ} / \mathrm{sec}$. A method of reproduction is used to estimate the perceived size of a moving figure. Twelve students serve as $S$ s. Possible bias owing to the method used is also measured. Four out of five stimuli used tend to show a similar tendency when possible bias is corrected. An equation showing the relationship between perceived size and moving speed is suggested.
\end{abstract}

It is well known that moving objects change their perceived shape and size when their moving speed increases. The senior author (1943a, 1943b, 1944) found that change of the perceived size of a moving figure is a function of both speed and original size of the figure when a black or bright rectangle projected on a screen moves horizontal direction. $\mathrm{He}$ also found that the important aspect of figural size related to its change in motion is the length of the rectangle parallel to the moving direction. The term "length of the figure" denotes the length parallel to the moving direction and "width of the figure" denotes its length parpendicular to the moving direction. The length of the figure is the most important factor in perceived size of a moving figure and the width of the figure has relatively small weight compared with the weight of the former.

The perceived length of a rectangular figure becomes shorter as the moving speed increases up to a certain speed, and then, it begin to increase its perceived length as the speed still increases in the case of a large figure whose length is relatively long. But in the case of small figure, whose length is relatively short, the perceived length of the figure begins to increase even under low speed. In the case of figures of intermediate size, they usually show very small change in spite of enormous change in moving speed. Morinaga, Noguchi and Ohishi (1963) confirmed the same tendency and named the decrement of the perceived length of a moving figure a reduction phenomenon. It is this reduction phenomenon we will deal with in the present study.

The change of the perceived size of a rectangular figure has two phases: the figure increases its length and the phase the figure decreases its length. The former may be related to the process of fusion. But there is no adequate explanation why a larger figure shows reduction phenomenon.

\section{EXPERIMENT I}

The purpose of the present experiment is to define quantitative relationship between the perceived length of a figure and its moving speed especially in the case the reduction phenomenon appears.

\section{Methods and procedures.}

The apparatus used in the present experiment is similar to the one used by the senior author in his previous experiments. The main 

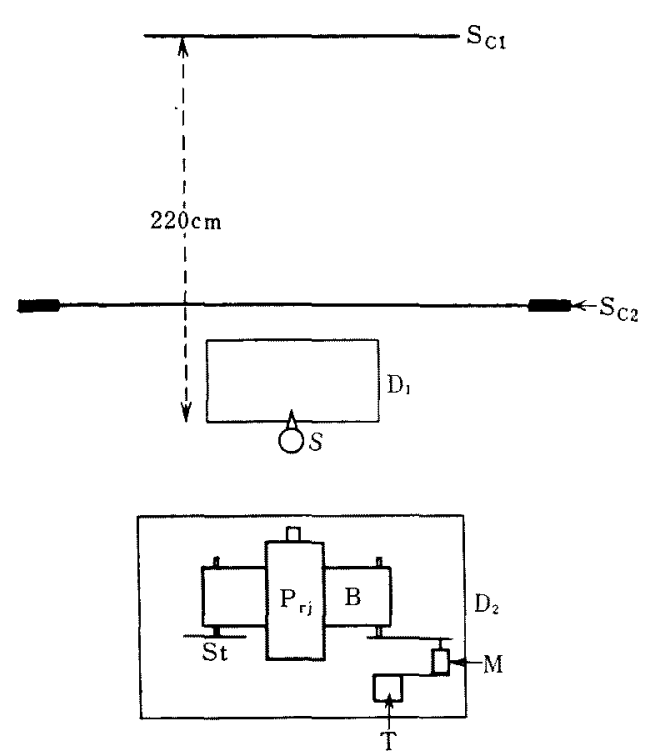

FIG. 1. Main arrangement of the apparatus. $\mathrm{S}_{\mathrm{c} 1}$ : screen where stimulus is projected. $\mathrm{S}_{\mathrm{e} 2}$ : screen with apertures. Prj: projector. B: belt. $\mathrm{M}$ : motor. St: stroboscope used to measure the speed.

arrangement of the apparatus is shown in Fig. 1. A black rectangular figure is attached to the center of a white belt which is driven by an electric motor, the speed of which can be changed by a transformer. The speed is measured by a stroboscope.

The stimulus figure is projected on a grey screen, $54 \mathrm{~cm} \times 54 \mathrm{~cm}$ in dimension, and the reflectance of which is $63 \%$. The figures used are rectangles the dimensions of which are $1.2 \mathrm{~cm} \times 2.4 \mathrm{~cm}, 2.4 \mathrm{~cm} \times 4.8 \mathrm{~cm}, 4.8 \mathrm{~cm} \times$ $9.6 \mathrm{~cm}, 9.6 \mathrm{~cm} \times 19.2 \mathrm{~cm}$ and $19.2 \mathrm{~cm} \times 38.4 \mathrm{~cm}$ on the screen. The moving speed can be changed from $124 \mathrm{~cm} / \mathrm{sec}$ to $465 \mathrm{~cm} / \mathrm{sec}$. Luminance of the figure on the screen is $0.043 \mathrm{~mL}$ and that of background is $0.795 \mathrm{~mL}$. Viewing distance is $220 \mathrm{~cm}$.

Subject sitting in a dark room is requested to fixate at the center of the screen when the light of the projector is on. Immediately after the light of the projector is on, a moving figure appears on the screen and moves horizontally to the left. Room light is turned on after the moving figure disappears and $S$ is requested to reproduce the shape of the seen figure.

Two methods of reproduction are used. In the first method, subject is requested to adjust black frames in order to reproduce the shape of the seen figure. In the second method, $S$ is requested to draw what he has seen on a sheet of white paper. No essential differences are found in the results obtained by these two methods although $S$ s usually report that the first method is easier compared with the second one. The results reported in this paper are based on the data obtained by the second method.

An index $z$ is used to show the perceived size of the stimulus, which can be caluculated by the following equation:

$$
z=(l / h) /(L / H)
$$

where $l$ denotes the length of the reproduced figure

$h$ denotes the width of the reproduced figure

$L$ denotes the length of the original figure

$H$ denotes the width of the original figure

$S$ s are 12 undergraduate students majoring in psychology, all of them have normal visual acuity or corrected normal. They are trained to the observation and the procedures in advance.

Results.

Results obtained are shown in Fig. 2. All of the figures used show the same tendency of reducing their perceived lengths when the moving speed increases. The results coincide with what we have anticipated as we have chosen those figures and speed which showed the reduction phenomena in the previous experiments.

Although these figures show the same tendency of reducing their perceived lengths as the moving speed increases, the amount of change differes from figure to figure. The stimuli may be classified into three groups according to the amount of reduction phenomena. The first group 


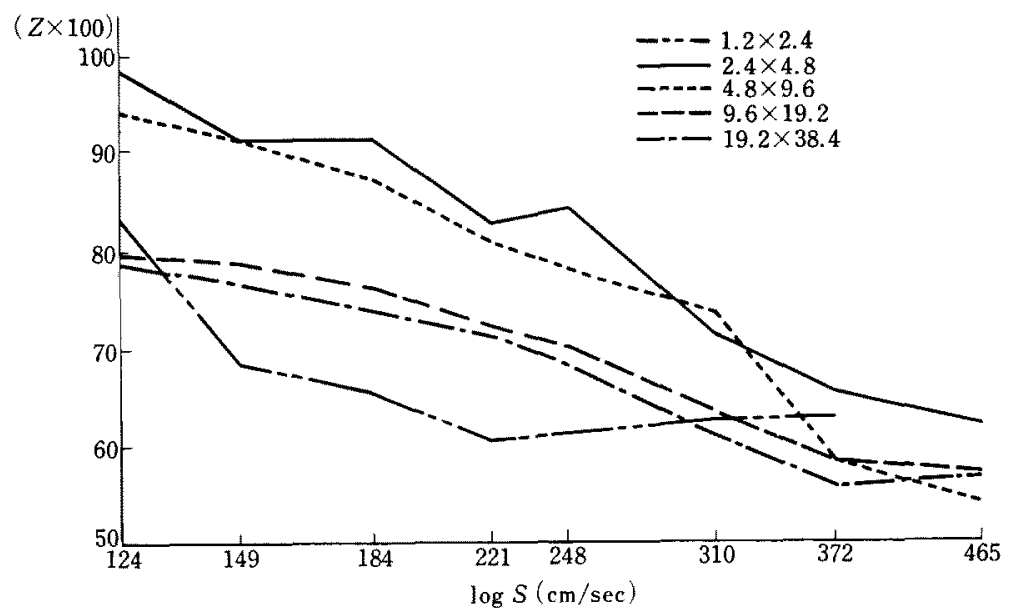

FIG. 2. Change of the length of moving figure with the change of moving speed.

may be consisted of two figures, $2.4 \mathrm{~cm} \times$ $4.8 \mathrm{~cm}$ and $4.8 \mathrm{~cm} \times 9.6 \mathrm{~cm}$. The second group may consist of also two figures, $9.6 \mathrm{~cm} \times 19.2 \mathrm{~cm}$ and $19.2 \mathrm{~cm} \times 39.4 \mathrm{~cm}$. The third group consists of only one figure, $1.2 \mathrm{~cm} \times 2.4 \mathrm{~cm}$, the smallest one. There are some irregularities in the data of the smallest figure as some of the $S_{\mathrm{s}}$ show somewhat different tendency. All of the $S \mathrm{~s}$ show the same tendency of reducing the length of the perceived figure when speed is relatively low, but some begin to show the tendency of increasing the length when the speed becomes relatively high.

There may be some doubt about the nature of the index $z$ as a true index of the perceived size of the figures. Although the shape of the figures used in the present experiment is the same, some bias may enter into the index $z$ owing to the method used. If the same index $z$ is used in the case of stationary figures, there may be some difference owing to the method used. Although we can not expect so large an illusion as is seen in $z$ under movement in this type of stationary figures, the index may differ owing to the difference of the size of the figures.

\section{EXPERIMENT II}

\section{Method and procedures.}

The five figures used in Experiment I are projected on the screen one by one and subject is requested to draw the shape of the seen figure on a sheet of white paper. The same index $z$ is used for calculating the perceived size of the figures. Three subjects are used.

Results

The results obtained are shown in Fig.



F1G. 3. Relationship between $z$ and size of figure. The size of the figure is given by linear dimension. 
TABLE 1

Corrected zs for five moving figures

\begin{tabular}{c|cccccccc}
\hline Fig. $\mathrm{cm} \times \mathrm{cm} / \mathrm{sec}$ & 124 & 149 & 184 & 221 & 248 & 310 & 372 & 465 \\
\hline $1.2 \times 2.4$ & .984 & .631 & .612 & .550 & .577 & .593 & .619 & - \\
$2.4 \times 4.8$ & .975 & .900 & .906 & .820 & .835 & .709 & .648 & .617 \\
$4.8 \times 9.6$ & .991 & .967 & .918 & .848 & .822 & .777 & .613 & .574 \\
$9.6 \times 19.2$ & .921 & .911 & .882 & .837 & .816 & .732 & .677 & .667 \\
$19.2 \times 38.4$ & .961 & .935 & .899 & .871 & .837 & .745 & .682 & .696
\end{tabular}

3. There is obviously a tendency that $z$ decreases as the size of the figure increases.

\section{Discussion}

The results of the Experiment II indicate that the index $z$ shows decrement as the size of the figure becomes large even in the case of stationary figure. Therefore, it is necessary to take the factor into consideration in order to decide the effects of moving speed on the perceived size of figure. An adequate solution of this problem is not easy as perceived size and shape of figure change owing to the movement of the stimulus. In order to find an adequate solution, it is necessary to expand the design of the Experiment II to cover all of the possible cases of shape and size of figure. But as a first approximation, we may be able to apply the results of the Experiment II to correct the possible effect of biases in the results of Experiment I. Such an application may partially be justified by a general tendency seen in the results of Experiment I where results of group 1 and 2 are roughly parallel. The corrected results are show in Table 1.

The corrected results show that Groups 1 and 2 coincide rather well. The results can be approximately shown by the following equation:

$$
z=-.625 \log v+2.295
$$

where $v$ denotes the speed of moving figures. The equation can not be applied to the results of the smallest figure in Experiment I. Its application is also limited to the range of speed used in the Experiment $I$.

\section{Summary}

The amount of decrement in the length of a rectangular figure is measured under different moving speed of the stimulus. The stimulus figure is projected on a screen and moves horizontally. A method of reproduction is used. Possible bias owing to the method used is also measured. Four out of five stimuli used show quite similar tendency when the possible bias is corrected.

\section{REFERENCES}

Morinaga, S., Noguchi, K., \& OHishi, A. 1963 Perception of shape and motion: On the reduction phenomena in perceptions of moving figures. $J$. Coll. Arts Lit. Chiba Univ., 4, 19-23.

TANAKA, Y. 1943a Perception of moving figures (1). Jap. J. Psychol. 17, 333-352.

TANAKA, Y. 1943b Perception of moving figures (2). Jap. J. Psychol., 17, 443-458.

TANakA, Y. 1944 Perception of moving figures (3). Jap. J. Psychol., 19, 1-11.

(Received Jan., 8, 1971) 